\title{
A short survey on next generation 5G wireless networks
}

\author{
Azra Yıldız ${ }^{*}$, Šejla Džakmić1 ${ }^{1}$ Mohammed Ahmed Saleh ${ }^{2}$ \\ ${ }^{1}$ Department of Computer Sciences and Engineering, International University of Sarajevo, Bosnia \\ ${ }^{2}$ Faculty of Electrical Engineering, University of Technology MARA (UiTM), Shah Alam, Selangor, Malaysia
}

*Corresponding author: amehic@ius.edu.ba

\section{(C) The Author}

2019.

Published by

ARDA.

\begin{abstract}
Current 4G - the fourth-generation wireless communication, which exists in most countries, represents an advance of the previous 3 generation wireless communication. However, there are some challenges and limitations, associated with an explosion of wireless devices, which cannot be accommodated by $4 \mathrm{G}$. Increasing the proliferation of smart devices, the development of new multimedia applications, and the growing demand for high data rates are among the main problems of the existing $4 \mathrm{G}$ system. As a solution, the wireless system designers have started research on the fifth-generation wireless systems. $5 \mathrm{G}$ will be the paradigm shift that could provide with ultra-high data rate, low latency, an increase of the base station capacity, and the improved quality of services. This paper is a review of the changes through the evolution of existing cellular networks toward 5G. It represented a comprehensive study associated with 5G, requirements for $5 \mathrm{G}$, its advantages, and challenges. We will explain the architecture changes - radio access network (RAN), air interfaces, smart antennas, cloud RAN, and HetNet. Furthermore, it discussed physical layer technologies, which include new channel modes estimation, new antenna design, and MIMO technologies. Also, it discussed MAC layer protocols. The article included three kinds of technologies: heterogeneous networks, massive multipleinput and output, and millimeter-wave. Finally, it explained the applications, supported by $5 \mathrm{G}$, new features, various possibilities, and predictions.
\end{abstract}

Keywords: 4G, 5G, Wireless Network, HetNet, MIMO, D2D, M2M, IoT, IoV

\section{Introduction}

The mobile wireless communication was introduced with the first generation - 1G. It was the voice-only system, initiated in the 1980 . However, it has been replaced by the second generation $-2 \mathrm{G}$, when the analog radio signals used by $1 \mathrm{G}$ were replaced by digital in the 1990 . The main feature of $2 \mathrm{G}$ was to provide delivering of the multimedia messages (text and picture messages) at low speed. In the recent time, people started to use the mobile phones in their daily lives and the demand for data services such as internet was increasing. So, there occurred a need for the technology to offer high speed data, which resulted in wireless communication evolving toward the third generation - 3G. Beside the hide speed data transmission, the main characteristics of the 3G were more security, video conferencing, 3D gaming, TV streaming, high bandwidth requirements, and large capabilities and so on. With the technology advancements, the smart devices popularity increasing and wireless communication were evolved toward fourth generation $-4 \mathrm{G}$. This, current generation, offers the same feature as $3 \mathrm{G}$, but also additional such: multimedia newspapers, watching TV programs and faster data transmission [1]. However, data traffic volume continues increasing, so it is predicted that it will grow up to 1000 times of the value in 2010 by year 2020 . The main reasons are: the

This work is licensed under a Creative Commons Attribution License (https://creativecommons.org/licenses/by/4.0/ ) that allows others to share and adapt the material for any purpose (even commercially), in any medium with an acknowledgement of the work's authorship and initial publication in this journal. 
proliferation of devices and new applications emerging. The researchers are working on exploring the next applications: Internet of Things (IoT), Internet of vehicles (IoV), Device to Device (D2D) communications, ehealthcare, Machine to Machine (M2M) communications and others [2]. Also, in order to enhance capacity and data rates, LTE cellular network is developing MIMO, small cells, HetNets and multiple antennas. All these, are the challenges of evolving toward fifth generation - 5G wireless communication. The main requirements of this generation are: increase in wireless data rates, reduction in trip latency, high bandwidth, large number of connected devices, reduction in energy consumption and high battery life. Those vision and challenges encourages us to write more detailed article about the next generation 5G wireless communication. In the rest of the paper, we will discuss about 5G architecture, physical layer, MAC layer and 5G applications.

\section{Literature Review}

In this part, we will mention the ideas of the three articles that contain discussion about the next generation of the wireless communication. For instance, Ning Zhang and others [2], state in their 'Cloud Assisted HetNets Toward 5G Wireless Networks' article that growth in mobile data traffic volume, almost doubles every year from 2010 by now. So, such venture leads forward to requirement for the next, more improved fifth generation in network communication. Also, according to Mamta Agiwal et al [3], one of the main role of the next generation $5 \mathrm{G}$ wireless is right to provide very high data rates. However, they also claim that its role is to provide high capacity, low latency and quality of service (QoS) improvement, compared to today's 4G. In their article 'Next Generation 5G Wireless Networks: A Comprehensive Survey', they offer very detailed discussion about $5 \mathrm{G}$ emerging reasons, vision and challenges. $5 \mathrm{G}$ technologies and emerging applications are very well explained by Tondare S.M et al, [4] in their journal 'Future of Mobile Communication 5G: Perspectives, Challenges and Services'.

\section{5G - A Paradigm Shift}

It is predicted that the architecture of the new network will be changed. There will be added new technologies to form faster network, which could deliver the rich traffic of multimedia and other data. 5G architecture requirements include advances in Radio Network, Air Interface, Smart Antenna, Cloud RAN and HetNets.

\subsection{Radio Network Evolution}

The researchers are working on the design of the device centric networking which will move the current Base Station centric network [3]. The 5G network also go forwards using of the higher frequency communication through mm-W signals. However, the propagation of this kind of signal has some limitations for the signal outdoor environment [5]. For example, for high data rate, a line of sight (LOS) communication has better performance over non-line of sight (NLOS). The solution is dual-mode modem which will provide the user to switch between more networks. So, mm-wave can be used for data communication, but control and system information can be transmitted using 4G. The narrow beams will improve link quality between BS grids and big number of users, so the $5 \mathrm{G}$ radio networking will be different a lot from the legacy networks. Its evolution will also affect the air interface.

\subsection{Advanced Air Interface}

$5 \mathrm{G}$ network communication provides the change from omni-directional to a directional antenna topology. Frequency reuse for beaforming antennas at both transmitter and receiver will be improved by using Spatial Division Multiple access (SDMA) [6]. However, there could be a problem to connect every antenna to high rate (A/D) and (D/A). Some possible solutions are the hybrid architecture and integrating analog and digital beamforming. Separation of the BS into different areas additionally relaxes the hardware, but it brings other difficulties in synchronization and information transmission. Optimal antenna configurations for various beamforming procedures improve execution. Huge BS arrangement and requirement for LOS communication can be facilitated by the partition of uplink and downlink. 


\subsection{Smart Antenna}

One of the key successes in 5G network development is an effective antenna array design. It relies on the progress of the air interface change. Smart antenna's role is to help in interference reduction, while in the same time maintain certain area and transmit power reduction of mobile handset and BS. Also, it obtains location information on all subscribers and increases the system capacity [7]. The use of the narrow beams results in more energy transmission at higher frequency. Among other advantages of smart antenna is also that same channel can be used by different beams. It reduces co-interference problem. Even weaker antennas can provide significant capacity gains [8]. That is why the design of the smart antenna is significant for 5G wireless communication. Smart antenna includes subarrays, which are important for beam steering. Those subarrays can be arranged in three different ways: (i) circular, (ii) planar and (iii) segmented. Circular subarray is meaningful for wireless communication, while curvature provides wider beam steering and better directivity of linear configuration. Segmented configurations can also achieve the required dimension of directivity and scan range. The horn antennas have the highest gains among all other antennas since its array provide high power output at BS [9].

\subsection{Centralized Architecture-Cloud RAN}

Cloud Radio Access Network (C-RAN) refers to the mobile network architecture that can address a various difficulties the operators face while try to support rising end-user's requirements. Actually, it tries to advance network architecture, mobility, performance, but also to reduce network costs. The significance of the C-RAN lies in solution of the problems related to increasing demands for high data rates [10]. Network industries are focused on network capacity improvements by more cells addition, designing MIMO techniques, adding more complex heterogeneous network structure. Nevertheless, these efforts are interrupted by CAPital EXpenditure and OPerating EXpenditure. The important characteristic of C-RAN is that it is based on centralization and virtualization. For example, it is intended for pooling the Baseband Units from more BS into the centralized one for multiplexing purpose. Remote Radio Heads (RRH) is contained from transreciever parts, amplifiers, duplexer digital process, A/D conversions power amplification and filtering. RRH is connected to BBU pool only with single mode fibre of data rate larger than $1 \mathrm{Gbps}$. Therefore, cloud computing ability can easily manage more complex control processes. Moving the RF to BBUs, results in radio frequencies to be generated in the BBU by itself. All transmissions are done by a mutual cloud-radio over fiber framework. It allows the use of analog RF, which further helps to services and operators to coexist with no major interference [11]. Researchers suggest SDN based virtual networks with the cloud in the role of the backone. It allows the connection of cloud applications with wireless networks through programmable interfaces [12]. Heterogeneous backhaul equipment which integrating the fixed broadband as well as wireless LOS backhaul is suitable as an infrastructure. Therefore, a standardized interface is used for both, designing and optimizing RAN along with backhaul network. RAN as a service (RANas), refers to the centralized cloud platform with both, packaging and delivering options. C-RAN enables shared pool of resources increasing deployment, management and operational efforts [13].

\subsection{Heterogeneous Approach-HetNets}

Heterogeneous Networks is composed of large number of small cells with varying transmission power. It refers to the big and vast network with different base stations (BS): macro, pico and femto using those smaller cells with low transmission power. By the setting of low power small BSs, network capacity is advanced and coverage is extended to cover indoor and outdoor. [6]. Radio Access Technology can be used to generate unnecessary signalling overhead, suggesting the use of an efficient multi-RAT handover [14]. A large number of frequency algorithms and reuse options improve heterogeneous network performance. Spectral resource arrangement is a potential solution for interference problem. It is proposed that the architecture of the 5G will replace the base station centric and import device centric networks, from small to femto cells which will form 
the heterogeneous network [6]. It is believed that heterogeneous connectivity of small cells is the main building block for 5G architecture [13].

\section{Physical layer design issues}

In order to apply $5 \mathrm{G}$ architecture over existing wireless systems we need a proper understanding for the physical layer and some of it is concepts like understanding how mm-wave channel works, adaptive Beamforming and Massive MIMO system which we are going to discuss in this part of the paper.

\subsection{Understanding mm-wave wireless channel}

The raise of the mm-wave brings new challenges in the communication systems. The first challenge we face is un-availability of any standard channel model beside that we need to care about the effects and biological safety of this waves [15]. For the technical part we need to do channel characterization looking at 4 different aspects/characteristics which are propagation loss, signal penetration, doppler and multipath [16]. Propagation loss of higher frequencies means prominent loss, where shorter wavelengths and directional transmission of narrow beams makes a higher and better performance of spatial multiplexing and reduces the interference Also, in order to have effective and good system design we need to study mm-wave propagation in different environments.

\subsection{Adaptive beamforming}

In this section we are going to discuss the importance of smart antennas for mm-waves and generally for the integration of $5 \mathrm{G}$ networks. Creating and Controlling the Beam: we need a good understanding of mm-waves if we want to focus the energy to wanted direction. We can apply Beamforming in digital, analog or RF forms[17]. comparing digital and analog beamforming we can see that digital preforms better with and higher complexity and cost on the other side we see the analog as an effective and simple method but as not flexible as digital one [17]. Antenna training protocols: it is obvious that the directional antennas are the future of 5G networks and as far as it is good it also brings new problem with it is design, beams are either good aligned and have a connection or beams are not aligned at all and accordantly not connected.

\subsection{Massive MIMO system}

Base station is provided by with a very large number of antennas with the help MIMO systems and linear signal processing methods, these antennas are able to send both vertical and horizontal beams [18]. Massive MIMO increases both spectral and energy efficiency. Each antenna is arranged in a way to achieve directivity during transmission [19]. 2D grid configuration and deployment of antenna arrays in massive MIMO systems are suggested. Also, 3D and distributed array structures could be candidate for 5G system.

\section{MAC layer}

In this part, needed changes in MAC protocol, multiple access, multiplexing and frame structure will be discussed.

\subsection{Spatial beam patterns}

A SDMA is a connector for adaptive antennas, beamforming and device centric $5 \mathrm{G}$ architecture. To support SDMA, base stations need to transmit and receive more beams in different directions, in the same time. Digital baseband beamforming plays the key role in such multiple beam transmission and receptions. A crucial need of SDMA is to enable adaptive antennas to direct the energy in the certain direction. One more important requirement is an estimation of direction and angle of arrival for RF connection between base station and mobile device. New MIMO SDMA-OFDM model is discussed in details in [20]. 


\subsection{Directional MAC protocols}

A MAC protocol could increase the network capacity. Multiuser MAC protocols are predicted to be based on directional Carrier Sense Multiple Access with Collision Avoidance [21]. Directional MAC protocol's importance lies in extension of the association beamforming training time. During that time, the stations perform initial beamforming training with access point. It enables the stations to conclude which neighbouring stations direction is the best. Another important role of the directional MAC protocol is to determine the best transmission direction using multi-directional Request to Send/Clear to Send (RTS/CTS) packets [22]. RTS/CTS can be transmitted to each beam to improve the work.

\subsection{Advanced multiple access techniques for 5G}

The large spectrum in mmWave encourages Carrier Aggregation to grow up the system Bandwidth. The mmWave channels consist wide bandwidth and small delay spread. It results in shorter Cyclic Prefix and wide subcarrier spacing. OFDM and single carrier FDM are suggested for mmWave Mobile Broadband system (MMB). Some of the main advantages of OFDM are flexibility and multiple bandwidth design. OFDM multiplexing can be used for analysing mobile broadband communication [5]. It is believed that OFDM is an effective for mm wave, but different user needs, Internet and low latency are great challenge. IDMA, as a kind of CDMA, uses special interleaves for user segregation [23]. Interleaves use less complex multiuser model at the receiver. Sparse code multiple access (SCMA) used both QMA mapping and spreading. It has better performance comparing to low density kind of CDMA. Generalized Frequency-Division Multiplexing (GFDM) and Filter Bank Multi-Carrier (FBMC) are also potential to overcome the idea of 5G [24]. The main concept of GFDM is a block frame transmission consisted of time slots and subcarriers. It enables low latency of 5G. FBMC is non-orthogonal and needs simple synchronization. Therefore, it provides signalling overheads reduction.

\subsection{Alternate technologies}

The main changes which come with new MAC features can be listed as: Random Access Channel (RACH), cognitive radio and efficient and novel MAC protocols. RACH is not compatible with orthogonality. Furthermore, the transmission from M2M and IoT cannot be avoided. Therefore, devices need to incorporate bulky synchronization processes of random access, intended for orthogonality. Cognitive radio is intended for resource utilization improvement. The existence of both licensed and unlicensed radio nodes on the same bandwidth is suggested [25]. An efficient and novel MAC protocols are crucial for utilization of the full duplex design. It potentiates nodes to send and receive a packet at the same frequency in the same time. The network throughput could be improved by Asymmetrical Duplex MAC protocol. It would establish dual link between half duplex clients and full duplex access point [26]. All these improvements in MAC layer technologies are predicted to bring large paradigm shift in the communication industry.

\section{5G Application}

5G technologies will make a great impact on future industries of all kinds, such as education, transportation, healthcare, entertainment, etc [27]. In fact, it will make a great global effect to the whole world society that will use benefits from it in every way. But it will also make us more dependable on our mobiles, too. Mobility will become an essential part of our lives without us even noticing, but it will bring us, says predictions, trillions of worth revenue in coming years.

\subsection{D2D communications}

In order to have device centric communication for $5 \mathrm{G}$, there is a need for fundamental changes of design and structure of the current cellular network. Device-to-device (D2D) communication [28] has been envisioned as an allied technology of $5 \mathrm{G}$ wireless systems for providing services that include live data and video sharing. A D2D communication technique opens new horizons of device-centric communications, i.e., exploiting direct 
D2D links instead of relying solely on cellular links [29]. Device-to-device communication enables two parties to communicate directly, without or with partial involvement of network infrastructure. This direct communication between nearby mobile devices will improve spectrum utilization, overall throughput and energy efficiency while enabling new peer to peer and location based applications and services [30]. Those are all vital aspects of $5 \mathrm{G}$ networks, and they work like this [31]:

- D2D communication can support local data services very efficiently through unicast, groupcast or broadcast transmission.

- Information sharing is well supported in a way that user can use D2D links to transmit files with higher data rates using low energy.

- Device with good Internet can be used as a hotspot.

\subsection{Internet of Things}

An IoT device is a simple piece of technology that has a small CPU, memory, display, communication and power and it is even more powerful when combined with large numbers of IoTs. Then, they can do streamline operations, enhance the security and safety of people as well as enabling efficient use of power and monitor our health [32]. In a nutshell we can say that IoT represents a millions of simultaneous connections, involving variety of devices, smart grids and transportation systems or we can say IoT is connecting anything, anyone, anytime and anyplace

5G offers some key differentiators in establishment of IoT. It represents a paradigm shift in architecture, multiplexing and networking, and it extends coverage, higher throughput and lower latency. Also, 5G presents a connection density of 1000x bandwidth per unit area, and 10-100x number of connections [33].

Six unique challenges of IoT [34]:

- Automated sensor configuration

- Context discovery

- Acquisition, modeling and reasoning

- Selection of sensors in 'sensing-as-a-service' model

- Security-privacy-trust

- Context sharing

More recently, Social Internet of Things (SIoT) is also coming up for exploring the relationship between objects and form social networks.

\subsection{Internet of Vehicles}

Development of IoT indubitably leads to the evolution of IoV. IoV represents a network of mutually linked vehicles for powerful traffic management which also reduces any possibility of any kind of crash. In response to this, 5G offers small cell cite specific environment for such a network which also offers dedicated links, content-centric network, cloud capabilities and low latency commitments [34]. The need for connected vehicle market arose from the need to be connected to anything anytime, so thanks to this, extremely large number of connected end points will exchange data. Vehicles would be given a potential to be connected to any physical thing as well as to humans, actions, goods, etc. in a completely reliable and secure way. Vehicles will not be only items with wheels that can take us places, but a whole new concept of living, servicing and delivering. Vehicles as complex as that are found as smart and interactive social object that form Social Internet of Vehicles (vehicular social networking platform). This platform has a cyber based architecture with cloud assisted data processing that helps this large network in traffic management. 


\subsection{Health care and wearable}

Today's critical health care services are craving for more reliable system. The report of Ramona Socher identified 3 emerging technologies as crucial to healthcare transformation:

5G New Radio interface and access extending beyond those of previous generations of mobile communication. The system will possess massive capacity, exceptionally low latency, ultra-high reliability and availability, and very low energy consumption. Federated Network Slicing enabling roaming of network slices in other networks to deliver global reach. Employing RAN virtualization and Distributed Cloud to ensure very low end-to-end latency. AI and real-time machine learning analytics will become crucial elements of making the networks self-optimizing to secure the fulfillment of service-level agreements. We will discuss only the first one stated. Wearable technology promises to provide health care solutions to growing world strained by the ballooning ageing population [35].

Appearance of $5 \mathrm{G}$ wireless together with Body Area Network (BAN) lift realtime monitoring of patient's health to the whole new level. This solution makes bandwidth constraints tough to reach, which in fact was the main problem with an old system. It also enables multiple physiological signals to be developed in specialized devices which will help to understand the disease pathophysiology. Medical system should be an IoT based system that will manage a big data on a cloud in order to have everything safe and in one place for the care of their patients, and be there for them anytime, anywhere, and this approach would make that happen. $5 \mathrm{G}$ remote is required to determine enormous information difficulties of health care applications conveying advantages to the humankind.

\subsection{M2M communication}

Machine-to-machine communication is a way of exchanging data between two machines without human interaction [36]. Wireless connection made M2M communication much easier and it allowed many more applications to be connected. Difference between M2M and IoT is in its network layer where M2M is one-toone communication while IoT is many-to-many communication network. Also, complexity of each of these is different in a way that M2M has one central monitoring system that tracks down operations and gives instructions to each of connected devices, while IoT has no such a thing. There each device can use rules and instructions from any place in a network.

M2M key point are:

- Massive number of devices

- Automated data generation and processing

- Relay station

- Converged mobile network

- Energy efficient

$5 \mathrm{G}$ can offer a large spectrum of features to $\mathrm{M} 2 \mathrm{M}$, such as, new mm-wave spectrum which can easily accommodate growth of devices. Besides, new waveforms could potentially answer odd traffic. 5G's low latency feature can resolve time critical issues and C-RAN and SDN architectures loosen tight coupling between the control planes and the data. To cope with the peculiar nature of M2M traffic, the evolving $5 \mathrm{G}$ system considers the integration of key enabling networking technologies for ubiquitous connectivity and guaranteed QoS.

\subsection{Miscellaneous applications}

Besides all these applications mentioned, we must not omit the greatest application of data processing in today's world - financial industry. 5G systems have a tremendous potential to change distinctive monetary administrations, like keeping money, installments, shared exchange and nearby business. Smart Grids are 
wireless networks for vitality information accumulation that control line observing, insurance and request/reaction management. High bandwidth and low latency of 5G can solve SG challenging demands [37]. The same is with smart homes with all its smart construction inside and out, and on the large scale, smart cities (traffic and energy management, emergency response, smart street lighting). The socio financial matters effect of 5G still cannot seem to be dissected. Be that as it may, it will have huge effect on each zone where remote transmission is unavoidable.

\section{Conclusion}

Next generation $5 \mathrm{G}$ nework connection is promissing a manifold growing up in data rate, connectivity and QoS. New applications, such are IoT, smart grids and IoV are predicted to be supported by $5 \mathrm{G}$ architecture. In this paper, 5G system is summarized. We began the discussion with 5G architecture, which refers to the design of radio network layout, smart antenna air interfaces and cloud and heterogeneous system. Also, we discudded about both, Physical and MAC layers and their improvments. Finally, we explained 5G architecture in details. We hope that this article will be usefull as a guedline for the future works in $5 \mathrm{G}$ wireless communications.

\section{References}

[1] M. L. J. Vora, "Evolution of Mobile Generation Technology: 1G To 5G and Review of Upcoming Wireless Technology 5G,” Sci. J. Impact Factor, vol. 2, no. 10, pp. 281-291, 2015.

[2] N. Zhang, N. Cheng, A. T. Gamage, K. Zhang, J. W. Mark, and X. Shen, "Cloud assisted HetNets toward 5G wireless networks," IEEE Commun. Mag., vol. 53, no. 6, pp. 59-65, 2015.

[3] A. R. and N. S. M. Agiwal, "Next Generation 5G Wireless Networks: A Comprehensive Survey," IEEE Commun. Surv. Tutorials, vol. 18, no. 3, pp. 1617-1655, thirdquarter, vol. 18, no. 3, pp. 1617-1655, 2016.

[4] Tondare S.M1, "Future of Mobile Communication 5G: Perspectives, Challenges and Services. In," IOSR J. Electron. Commun. Eng., vol. 9, no. 6, pp. 45-49, 2014.

[5] T. S. Rappaport, Y. Xing, G. R. MacCartney, A. F. Molisch, E. Mellios, and J. Zhang, "Overview of Millimeter Wave Communications for Fifth-Generation (5G) Wireless Networks-With a Focus on Propagation Models," IEEE Trans. Antennas Propag., vol. 65, no. 12, pp. 6213-6230, 2017.

[6] M. A. Al-Namari, A. M. Mansoor, M. Yamani, and I. Idris, "A Brief Survey on 5G Wireless Mobile Network,” IJACSA) Int. J. Adv. Comput. Sci. Appl., vol. 8, no. 11, pp. 52-59, 2017.

[7] Z. Feng and Z. Zhang, "Dynamic spatial channel assignment for smart antenna," Wirel. Pers. Commun., vol. 11, no. 1, pp. 79-87, 1999.

[8] P. Cardieri and T. S. Rappaport, "Application of narrow-beam antennas and fractional loading factor in cellular communication systems,” IEEE Trans. Veh. Technol., vol. 50, no. 2, pp. 430-440, 2001.

[9] C. F. Lai, R. H. Hwang, H. C. Chao, M. M. Hassan, and A. Alamri, "A buffer-aware HTTP live streaming approach for SDN-enabled 5G wireless networks," IEEE Netw., vol. 29, no. 1, pp. 49-55, 2015.

[10]A. Checko et al., "Cloud RAN for Mobile Networks - A Technology Overview," IEEE Commun. Surv. Tutorials, vol. 17, no. 1, pp. 405-426, 2015.

[11]A. Checko, "Cloud radio access network architecture. Towards 5G mobile networks," 2016.

[12] M. Banikazemi, D. Olshefski, A. Shaikh, J. Tracey, and G. Wang, "Meridian: An SDN platform for cloud network services,” IEEE Commun. Mag., vol. 51, no. 2, pp. 120-127, 2013.

[13] "THE NEXT GENERATION OF EUROPEAN The European telecommunications industry has been at the forefront of global competition ever since the early days of mobile," p. 196994, 2020.

[14] S. Talwar, D. Choudhury, K. Dimou, E. Aryafar, B. Bangerter, and K. Stewart, "Enabling Technologies and Architectures for 5G Wireless," 2014. 
[15]C. M. C. T. Wu, T. S. Rappaport, "The Human Body and Millimeter-Wave Wireless Communivcation Systems," no. Icc, 2015.

[16]T. S. Rappaport, Y. Qiao, J. I. Tamir, J. N. Murdock, and E. Ben-Dor, "Cellular broadband millimeter wave propagation and angle of arrival for adaptive beam steering systems (invited paper)," RWW 2012 - Proc. IEEE Radio Wirel. Symp. RWS 2012, pp. 151-154, 2012.

[17]C. Scarborough, K. Venugopal, A. Alkhateeb, and R. W. Heath, "Beamforming in Millimeter Wave Systems: Prototyping and Measurement Results," pp. 1-5, 2018.

[18] L. Lu, G. Y. Li, A. L. Swindlehurst, A. Ashikhmin, and R. Zhang, "An overview of massive MIMO: Benefits and challenges,” IEEE J. Sel. Top. Signal Process., vol. 8, no. 5, pp. 742-758, 2014.

[19] Y. Mehmood and W. Afzal, "Large scaled multi-user MIMO system so called massive MIMO systems for future wireless communication networks," 2013 19th Int. Conf. onAutomation Comput., no. September 2013, pp. 13-14, 2013.

[20] M. Jiang and L. Hanzo, "Multiuser MIMO-OFDM for next-generation wireless systems," Proc. IEEE, vol. 95, no. 7, pp. 1430-1469, 2007.

[21]M. X. Gong, D. Akhmetov, R. Want, and S. Mao, "Multi-user operation in mmwave wireless networks," IEEE Int. Conf. Commun., 2011.

[22]D. Wong, Q. Chen, and F. Chin, Directional Medium Access Control (MAC) Protocols in Wireless Ad Hoc and Sensor Networks: A Survey, vol. 4, no. 2. 2015.

[23]P. Niroopan and Y. Chung, "A User-Spread Interleave Division Multiple Access System," vol. 1, no. 10, pp. 837-841, 2012.

[24]N. Michailow, R. Datta, S. Krone, M. Lentmaier, and G. Fettweis, "Generalized Frequency Division Multiplexing: A Flexible Multi-Carrier Modulation Scheme for 5th Generation Cellular Networks," IEEE Trans. Commun., vol. 62, no. 9, pp. 3045-3060, 2012.

[25] I. Engineering, "Delay Minimization in Cognitive Mesh Networks Using Recursive Algorithm in DORP Protocol,” pp. 4672-4681, 2015.

[26] M. Kobayashi, R. Murakami, S. Saruwatari, and T. Watanabe, "Wireless Full-duplex Medium Access Control for Enhancing Energy Efficiency,” vol. X, no. X, pp. 1-30, 2017.

[27] S. Bergren, "Design Considerations for a 5G Network Architecture Steven," Acta Sci. Math., vol. 78, no. 3-4, pp. 643-667, 2012.

[28]F. Boccardi, R. Heath, A. Lozano, T. L. Marzetta, and P. Popovski, "Five disruptive technology directions for 5G,” IEEE Commun. Mag., vol. 52, no. 2, pp. 74-80, 2014.

[29]A. Asadi, Q. Wang, and V. Mancuso, "A survey on device-to-device communication in cellular networks," IEEE Commun. Surv. Tutorials, vol. 16, no. 4, pp. 1801-1819, 2014.

[30]K. Shen and W. Yu, "FPLinQ: A cooperative spectrum sharing strategy for device-to-device communications," IEEE Int. Symp. Inf. Theory - Proc., pp. 2323-2327, 2017.

[31] J. M. B. Da Silva, G. Fodor, and T. F. Maciel, "Performance analysis of network-assisted two-hop D2D communications,” 2014 IEEE Globecom Work. GC Wkshps 2014, no. July, pp. 1050-1056, 2014.

[32] V. Kumar, "Ubiquitous Data Accessing - Internet of Things in Healthcare Information System," Ijarse, vol. 4, no. 4, pp. 1097-1101, 2015.

[33]C. Perera, A. Zaslavsky, P. Christen, and D. Georgakopoulos, "Context aware computing for the internet of things: A survey,” IEEE Commun. Surv. Tutorials, vol. 16, no. 1, pp. 414-454, 2014.

[34] A. M. Ortiz, D. Hussein, S. Park, S. N. Han, and N. Crespi, "The cluster between internet of things and social networks: Review and research challenges,” IEEE Internet Things J., vol. 1, no. 3, pp. 206-215, 2014.

[35]D. Communication, "Wearable technologies and its future applications," no. 4, pp. 45-50, 2015.

[36] Y. Liu and S. Xie, "Cognitive Machine-to-Machine Communications: Visions and Potentials for the Smart Grid," no. June, pp. 6-13, 2012. 
[37]D. T. A. Ye Yan, Yi Qian, Hamid Sharif, "A Survey on Smart Grid Communication Infrastructures:," vol. 3994, no. September, pp. 1-16, 2015. 